\title{
Importance of Patient Involvement in the Value Assessment Process: On the Way Towards Personalised Treatments
}

\author{
Nicole Gusset ${ }^{1,2}$ \\ Accepted: 28 November 2021 / Published online: 16 December 2021 \\ (C) The Author(s), under exclusive licence to Springer Nature Switzerland AG 2021
}

\section{Introduction}

One concern around the widespread implementation of value assessment frameworks is the absence of the patient perspective [1,2]. Assessments meant to determine the value of new technologies could be missing crucial elements that define what it is like to live with a life-changing disease. Patients bring to the table their unique lived experience of specific conditions and of their care and medication, and are motivated to engage. The interest in engaging with patients across the cycle of research and development of medicines is high, but many stakeholders still do not know how to engage meaningfully and how to expand relationships from information provider to co-creator in healthcare. Usually, it requires time to move from a traditional sporadic one-way communication, tick-box exercise to a continuous partnership at eye-level with early involvement in relevant decision-making processes.

\section{Who is 'the Patient'?}

In one way or another, we are all 'patients'. So, why do we need a new stakeholder at the table that adds a perspective we are all supposedly familiar with? Usually, in a discussion, an individual can only take one position and wearing two hats at the same time will naturally cause conflicts of interest. Therefore, it is key for patients to be represented with an additional voice. Importantly, in the EUPATI guidelines [3], the term 'patient' is defined. Here, 'individual patients' are persons with personal experience of living with a disease; their main role is to contribute with their subjective disease and treatment experience. 'Patient experts', in

Nicole Gusset

nicole.gusset@sma-europe.eu

SMA Europe, Freiburg im Breisgau, Germany

2 SMA Schweiz, Heimberg, Switzerland addition to having disease-specific expertise, have technical knowledge in research and development and/or regulatory affairs through training or experience. Often they also bring a different perspective that encourages new approaches and ideas. Consequently, patient experts are an important strategic partner. In the value assessment process, their knowledge complements clinical research, helps to overcome uncertainties in clinical evidence, provides insights into care pathways, explains the benefits and challenges of using existing health technologies and shares experience and expectations of new technologies (e.g. [4-6]).

\section{Early and Continuous Involvement to Ensure Patient Relevance}

To date, there seems to be a disproportionate focus on clinical outcomes in value assessments, and value frameworks often take a population-level reimbursement perspective focussed on clinical outcomes and payer costs. Value assessments may thus disregard the evaluation of criteria important to patients at the individual level $[1,4,7]$. This could be addressed by early and regular involvement of patient experts in the different phases of the life cycle of medicines-and also when it comes to their value assessment [2]. More specifically, this might lead to an adjustment in the endpoints assessed in clinical trials and a shift away from primarily focussing on clinical outcomes and on populationlevel reimbursement perspectives.

1. Assessment of Clinical Trial Endpoints

Decisions taken in the design of clinical trials regarding the assessed endpoints and eligibility criteria will directly impact the understanding of the value of a product later; especially because value assessments tend to focus on selected subsets of clinical outcomes because these were the endpoints studied in trials [8-10]. Today, patient-reported outcomes are often generic and miss- 
ing patient relevance for a specific disease environment. Thus, early involvement of patients will ensure that patient-relevant measures are selected as endpoints. This is especially important because endpoints, but also inclusion criteria, eventually influence decisions regarding cost coverage [11] and may lead to subpopulations being excluded from access to treatment.

In spinal muscular atrophy (SMA), endpoints are predominantly clinical outcomes and not necessarily patient-relevant. Outcomes focus heavily on motor function as health states $[9,12]$ and tend to neglect other aspects, such as performing daily activities, fatiguability, fatigue, pain, psychological issues, or swallowing, which seem to be impacting wellbeing in patients $[13,14]$. Similarly, other meaningful motor-functional changes, such as fine finger movements that allow better communication via electronic devices, are not captured by current scales [15].

\section{Shift Focus Away From Clinical Outcomes}

So far, in many clinical trials, and especially in rare diseases, patients have not been involved early in the process, nor were there validated patient-relevant outcome measures available at the start of the trial. A change in focus in the value assessment is key. Only by involving patient experts, and by moving from purely assessing clinical outcomes towards including other values in the analyses that assess the impact on daily life, can patient relevance be ensured $[4,16]$. Unfortunately, today, only very few value assessment frameworks explicitly account for the patient perspective by including decision-making attributes that matter most to them. These are, for example, patient-reported outcomes, treatment-related attributes, resource availability and use and societal impacts (independence, family spill-overs, productivity, caregiver burden), but also the value of 'hope' [4]. In personal impact stories, such attributes have been collected [16]. The weighting of them can vary greatly between individual patients based on small differences in individual characteristics and experiences [8]. However, in order to sustainably include these attributes that go beyond traditional value assessment, these data must be collected systematically, including in the real-world setting and with patient involvement.

In SMA, the challenges we face in value assessments are typical for the field of rare diseases; specific instruments are not available and tailored methods are missing $[9,17,18]$. Particularly challenging are heterogenous and often non-transparent processes with lack of patient expert involvement, the lack of data about what attributes matter most to patients, and as a result the missing knowledge of the involved actors in this regard, limited life history data, lack of validated and disease-specific methods to assess quality of life, especially in the paediatric population, small study sample sizes and lack of long-term clinical data across different subpopulations (see also [9]).

Patient advocacy efforts have already underpinned the value of stabilisation, with specific and detailed patient input from daily life experience [19]. Stabilisation stops the progression of the disease, so people do not lose more life-years, but importantly also gives them a change in perspective and hope, directly positively impacting their quality of life. Importantly, quality-oflife gains seem to be strongly valued by the most disabled people, even more so than life-year extensions [20]. This, in turn, emphasises the life-changing importance of stabilisation.

3. Shift Focus Away From Population-Level Reimbursement

Traditionally, treatments are reimbursed on a (sub-) population level. However, the importance of patientcenteredness as a key value is becoming increasingly apparent. Importantly, as scientific advances permit greater personalisation when treating complex diseases, it is critical that value assessment frameworks strike a balance between decision making at population and individual levels [1]. This individual-focussed and more precise approach is especially important in rare diseases. Often, the narrow eligibility criteria in clinical trials lead to a homogeneous study population, but at the same time exclude many individuals. Only relying on data collected under such strict inclusion conditions inevitable leads to a knowledge gap, and consequently to a situation in which individuals are eventually left behind without the prospect of any treatment. Early and continuous involvement of patient experts helps to proactively identify such challenges on the one hand, and jointly find the best possible solutions for the patient community on the other.

In SMA, we are facing (sub-)population-level value assessments and reimbursement decisions that are not capturing the full picture of this spectrum disease. This has caused many inequalities in our community and led to enormous distress [19]. The implementation of a revised SMA classification, which does not purely rely on the diagnosed type but reflects the current mobility status [19], is a relevant change, but still has its limitations in reflecting reality. Overall, the artificial classification into subpopulations, such as type, mobility status and age, is challenging because within-group differences are sometimes larger than between-group differences. 


\section{Patient Relevance and Personalised Value Assessments}

Why are we holding on to population-level approaches in medicines development while at the same time claiming the era of personalisation? Why are we still dividing heterogeneous groups of people into subgroups instead of treating individuals according to their personal needs? Why do we not find ways to work with the same scientific quality at the level of the individual, and by doing so also moving the regulations and process frameworks of value assessment into the field of personalised medicines?

As scientific knowledge increases, diseases are divided into smaller and smaller subunits, and therapies are developed that work with increasing precision. At the same time, this seems to make value assessment, especially beyond clinical outcomes, increasingly complex based on fewer and fewer case-specific data. But already today, value can be assessed and modelled from different perspectives, such as society, disease and population or subpopulation level. Ideally, relevant data are collected along the life cycle of treatments, leading to a situation in which the most effective therapy for an individual is identified by using individual endpoints.

SMA has gained tragic notoriety because the most expensive therapies that have come to market to date are for SMA. This has triggered an avalanche of discussion about values and worth, leading to the question of how much a human life should cost. SMA as a rare disease serves as a prime example to illustrate that (i) approaches to value assessments in Europe are heterogeneous, leading to patchwork access to medicines, and (ii) there is a high need for personalisation. There are efforts to harmonise value assessments in Europe, but this cannot be done at the expense of the individual-on the contrary, transparent frameworks informed by the needs, preferences and experiences of individual patients should established. These will enable global data sharing, access to archives on identified patient-relevant value elements and the collection of additional data on value in order for them to be continuously refined. In this way, personalised treatment might become a reality, but only with serious and continuous involvement of patient experts.

Acknowledgments I would like to thank Yasemin Erbas and Markus Gusset for commenting on the manuscript.

\section{Declarations}

Conflicts of interest/competing interests N.G. is a volunteer for SMA Europe and SMA Schweiz, mother of a child living with SMA, advisor and lecturer for Biogen, Clinigen, Novartis Gene Therapies, Novartis and Roche.
Disclosure statement This editorial is published in a special edition journal supplement wholly funded by F. Hoffmann-La Roche Ltd.

Availability of data and material Not applicable.

Code availability Not applicable.

Author contributions Not applicable.

Ethics approval Not applicable.

Consent to participate Not applicable.

Consent for publication Not applicable.

\section{References}

1. Diaby V, Ali AA, Montero AJ. Value assessment frameworks in the United States: a call for patient engagement. PharmacoEcon Open. 2019. https://doi.org/10.1007/s41669-018-0094-z.

2. Wale JL, Thomas S, Hamerlijnck D, Hollander R. Patient and public are important stakeholders in health technology assessment but the level of involvement is low-a call to action. Res Involve Engage. 2021. https://doi.org/10.1186/s40900-020-00248-9.

3. Hunter A, Facey K, Thomas V, Haerry D, Warner K, Klingmann I, May M, See W. EUPATI guidance for patient involvement in medicines research and development: health technology assessment. Front Med. 2018. https://doi.org/10.3389/fmed.2018.00231.

4. Bohannon L, Franklin E. Value Assessment that puts patients at the forefront. Value Outcomes Spotlight. 2021;7(1):29-31.

5. Norburn L, Thomas L. Expertise, experience, and excellence. Twenty years of patient involvement in health technology assessment at NICE: an evolving story. Int J Technol Access Health Care. 2021. https://doi.org/10.1017/S0266462320000860.

6. Facey KM, Bedlington N, Berglas S, Bertelsen N, Single ANV, Thomas V. Putting patients at the centre of healthcare: progress and challenges for health technology assessments. The patientpatient-centered outcomes research. Prog Challenge Health Technol Access. 2018. https://doi.org/10.1007/s40271-018-0325-5.

7. Perfetto EM, Oehrlein EM, Boutin M, Reid S, Gascho E. Value to whom? The patient voice in the value discussion. Value Health. 2017. https://doi.org/10.1016/j.jval.2016.11.014.

8. Cannon E. Putting the patient voice first: Novel approaches to incorporating patient-centered outcomes in value assessment. Value Outcomes Spotlight. 2021;7(2):3-5.

9. Paracha N, Hudson P, Mitchell S, Sutherland CS. Systematic literature review to assess economic evaluations in spinal muscular atrophy (SMA). Pharmacoeconomics. 2021. https://doi.org/10. 1007/s40273-021-01095-6.

10. Kruizinga MD, Stuurman FE, Groeneveld GJ, Cohen AF. The future of clinical trial design: the transition from hard endpoints to value-based endpoints. In: Barrett J, Page C, Michel M, editors. Concepts and principles of pharmacology. Handbook of experimental pharmacology. Cham: Springer; 2019. p. 260. https://doi. org/10.1007/164_2019_302

11. Swift B, Jain L, White C, Chandrasekaran V, Bhandari A, Huges DA, Jadhav PR. Innovation at the intersection of clinical trials and real-world data science to advance patient care. Clin Transl Sci. 2018. https://doi.org/10.1111/cts.12559.

12. Lo SH, Gorni K, Sutherland CS, et al. Preferences and utilities for treatment attributes in type 2 and non-ambulatory type 3 spinal 
muscular atrophy in the United Kingdom. Pharmacoeconomics. 2021. https://doi.org/10.1007/s40273-021-01092-9.

13. Gusset N, Erbas Y, de Lemus M. Impact of fatigue, pain and psychological problems on perceived quality of life in pediatric SMA patients. Neuropediatrics. 2021. https://doi.org/10.1055/s-00411739574.

14. McGraw S, Qian Y, Henne J, Jarecki J, Hobby K, Yeh WS. A qualitative study of perceptions of meaningful change in spinal muscular atrophy. BMC Neurol. 2017. https://doi.org/10.1186/ s12883-017-0853-y.

15. Pierzchlewicz K, Kepa I, Podogrodzki J, Kotulska K. Spinal muscular atrophy: the use of functional motor scales in the era of disease-modifying treatment. Child Neurol Open. 2021. https:// doi.org/10.1177/2329048X211008725.

16. Single A, Facey K, Livingstone H, Silva A. Stories of patient involvement impact in health technology assessments: a discussion paper. Int J Technol Assess Health Care. 2019. https://doi. org/10.1017/S0266462319000552.

17. Pearson SD, Thokala P, Stevenson M, Rind D. The effectiveness and value of treatments for spinal muscular atrophy. J Manag
Care Spec Pharm. 2019. https://doi.org/10.18553/jmcp.2019.25. 12.1300.

18. Nestler-Parr S, Korchagina D, Toumi M, Pashos CL, Blanchette C, Molsen E, Morel T, Simoens S, Kalo Z, Gatermann R, Redekop W. Challenges in research and health technology assessment of rare disease technologies: report of the ISPOR rare disease specialist interest group. Value Health. 2018. https://doi.org/10. 1016/j.jval.2018.03.004.

19. Gusset N, Stalens C, Stumpe E, Klouvi L, Mejat A, Ouillade MC, de Lemus M. Understanding European patient expectations towards current therapeutic development in spinal muscular atrophy. Neuromusc Disord. 2021. https://doi.org/10.1016/j.nmd. 2021.01.012.

20. Lakdawalla DN, Phelps CE. Generalized Risk-Adjusted CostEffectiveness (GRACE): ensuring patient-centered outcomes in healthcare decision making. Value Outcomes Spotlight. 2021;7(2):5-10. 